seem to be something very different from definitions of words. Kant considers $7+5=12$ to be a synthetical judgment (Proleg., pp. 22, 23).

Metaphysics and mathematics are consorts in the East as well as the West. Bhascara says that the analytical art is merely sagacity exercised, and is independent of symbols, which do not constitute the art. $^{2}$ If De Morgan ${ }^{2}$ be right in placing Dioplantus as late as the beginning of the seventh centary, Aryabhatta was earlier, by two centuries, than Diophantus. The name, certainly, seems to have been a very common one. Josephus ${ }^{3}$ relates that Alexander (a sou of Herod the Great) said that Diophantus the scribe had imitated his hand. But Mr. Heath's work ${ }^{4}$ renders it scarcely possible to sustain Do. Morgan's contention.

On Raabe's Bernoullians. By J. D. Hamilton Dickson, M.A.

[Read Nov. 8th, 1888.]

Rasbe has given the name Bernoullian to the function

$$
\phi(z, m)=z^{m}+m_{1} B_{0} z^{m-1}+m_{8} B_{1} z^{m-2}+m_{8} B_{2} z^{m-8}+\ldots,
$$

where there is no term without $z ; m_{1}, m_{9}, \ldots$ being the successive Binomial coefficients after the first in the expansion of $(l+t)^{m}$, and $L_{\iota}, B_{1}, \ldots$ being Bernoulli's numbers, viz.,

$$
\begin{gathered}
B_{0}=-\frac{1}{2}, \quad B_{9}=B_{4}=B_{0}=\ldots=B_{2 n}=\ldots=0, \\
B_{1}=\frac{1}{6}, \quad B_{8}=-\frac{1}{30}, \quad B_{6}=\frac{1}{4}, \quad B_{7}=-\frac{1}{80}, \quad B_{0}=\frac{5}{86}, \quad \& \mathrm{c} .
\end{gathered}
$$

1 Colebroke, "Algebra," etc. (London, 1817), p. xix.

"De Morgan, "Arithmetical Books" (London, 1847), p. 47.

3 Josephus, "Antiquities of tho Jews" (Burder's 'Translation, Vol. 1., pp. 616, 617). IBurder's Proface is dated London, Octubur $1,1811$.

"'I. L. Ieath, "Diophantos of Alexandria: a Study in the History of Greek Algebra" (Cambridge University Press, 1885). 
For instance, the first Bernoullians are

$$
\begin{aligned}
& \phi(z, 1)=z, \\
& \phi(z, 2)=z^{2}-z, \quad=u \text { (say), } \\
& \phi(z, 3)=z^{3}-\frac{8}{2} z^{9}+\frac{1}{2} z, \\
& \phi(z, 4)=z^{4}-2 z^{8}+z^{2}, \\
& \phi(z, 5)=z^{5}-\frac{5}{2} z^{4}+\frac{8}{3} z^{3}-\frac{1}{6} z, \\
& \phi(z, 6)=z^{6}-3 z^{5}+\frac{5}{2} z^{4}-\frac{1}{2} z^{8}
\end{aligned}
$$

\&c. \&c.

Jacobi has shown [Crelle, Bd. xII. (1834), p. 271], and it is already well known, that

$$
\begin{aligned}
& \phi(z, 2 n-1)=\phi(z, 3) \cdot F(u), \\
& \phi(z, 2 n)=\phi(z, 4) \cdot G(u),
\end{aligned}
$$

where $F(u), G(u)$ are functions of $u$ of degree $n-2$. It might, therefore, be inferred that $F(u)$ and $G(u)$ might be expressed in terms of Bernoullians not higher than the $(2 n-4)$ th. The object of the present paper is to prove this and find the development.

Writing

$$
\phi(z, 2 n-1)=\phi(z, 3)\left\{a_{0} z^{2 n-4}+a_{1} z^{2 n-8}+\ldots+a_{r} z^{2 n-r-4}+\ldots+a_{2 n-1}\right\}
$$

and noting that

$$
\begin{aligned}
& \phi(z, 3)= z^{8}-\frac{3}{2} z^{9}+\frac{1}{2} z, \\
& \begin{aligned}
\phi(z, 2 n-1)=z^{2 n-1}+(2 n-1) B_{0} z^{2 n-2} & +(2 n-1)_{8} B_{1} z^{2 n-8} \\
& +(2 n-1)_{3} B_{2} z^{2 n-4}+\ldots
\end{aligned}
\end{aligned}
$$

on expanding the right-hand side of (1), and equating coefficients of powers of $z$, we get the equations

$$
\begin{aligned}
a_{r}-\frac{3}{2} a_{r-1}+\frac{1}{2} a_{r-2} & =(2 n-1)_{r} \quad B_{,-1}, \\
a_{r-1}-\frac{8}{2} a_{r-2}+\frac{1}{2} a_{r-8} & =(2 n-1)_{r-1} B_{r-2}, \\
\cdots \quad \cdots \quad \cdots \quad & \cdots \quad \cdots \quad \cdots \\
a_{2}-\frac{3}{2} a_{1}+\frac{1}{2} a_{0} & =(2 n-1)_{2} B_{1}, \\
a_{1}-\frac{3}{2} a_{0} & =(2 n-1) \quad B_{0}, \\
a_{0} & =1 .
\end{aligned}
$$


Hence, tho value of $a_{r}$ may be expressed ns a determinant of the $(r+1)^{\text {th }}$ order, viz., after a slight simplitication,

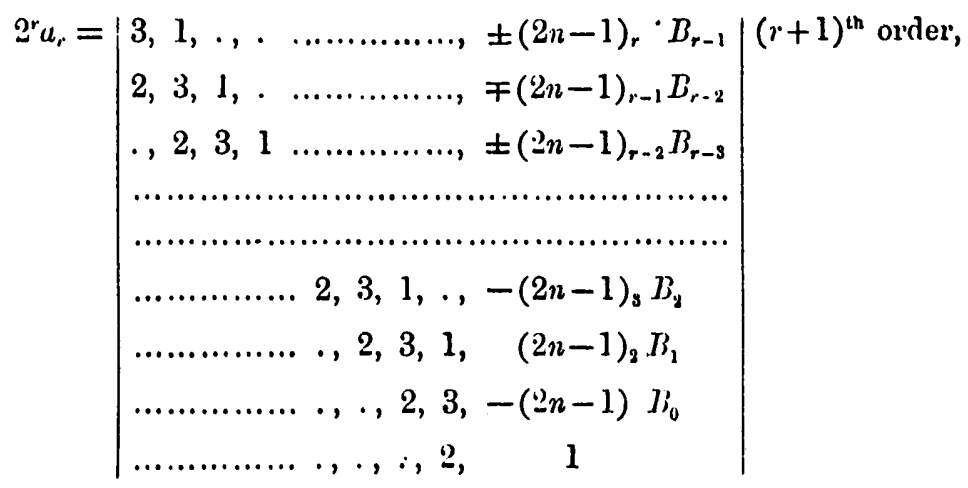

the signs of the terms in the last columu boing alternately + and -, logimning with the lowest term as + . 'This is casily ovaluated, and grives

$$
\begin{aligned}
2^{r} a_{r}=\left(2^{r+1}-1\right) & +2\left(2^{r}-1\right)(2 n-1) B_{0}+2^{2}\left(2^{r-1}-1\right)(2 n-1)_{2} B_{1} \\
& +2^{3}\left(2^{r-2}-1\right)(2 n-1)_{3} B_{2}+\ldots \\
\ldots+ & +2^{r-1}\left(2^{8}-1\right)(2 n-1)_{r-1} B_{r-2}+2^{r}(2 n-1)_{r} B_{r-1} \ldots(2) .
\end{aligned}
$$

Similarly, writing

$$
\phi(z, 2 n)=\phi(z, 4)\left\{b_{0} z^{2 n-4}+b_{1} z^{2 n-6}+\ldots+b_{1} z^{2 n-r-4}+\ldots+b_{2 n-4}\right\} \ldots(3) ;
$$

and noting that

$$
\begin{aligned}
& \psi(z, 4)=z^{4}-2 z^{3}+z^{2} \\
& \phi(z, 2 u)=z^{2 n}+2 n I_{v_{3}} z^{2 n-1}+(2 u)_{2} B_{1} z^{2 n-2}+(2 u)_{3} B_{8} z^{3 n-3}+\ldots ;
\end{aligned}
$$

on expimiling the right-hamd side of (3), and cquating cocflicients of jowers of $z$, wo get tho expuations

$$
\begin{aligned}
& b_{r}-2 b_{r-1}+b_{r-2}=(2 u)_{r}, b_{r-1}, \\
& l_{12}-m_{l_{1}}+b_{0}=\left(2{ }_{1}\right)_{2} l_{1} \text {, } \\
& b_{1}-\ddot{l}_{10}=\left(\ddot{\prime \prime}_{11}\right) b_{11}, \\
& l_{u}=1 \text {, }
\end{aligned}
$$


whence, as before,

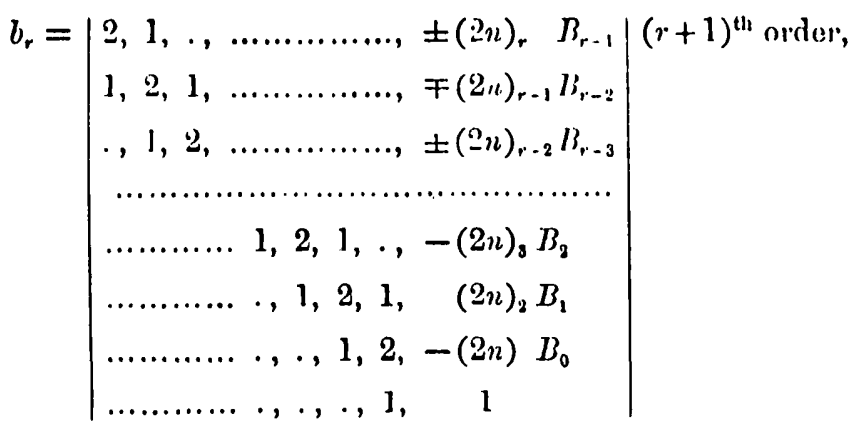

the signs of the terms in the last column heing ngain nlternately + and - cominencing with the lowest as + , an.l reading upwards.

Finally,

$$
\begin{aligned}
& b_{r}=(r+1)+r(2 n) B_{0}+(r-1)(2 n)_{2} B_{1}+(r-2)(2 n)_{8} B_{2}+\ldots \\
& \ldots+2(2 n)_{r-1} l l_{r-2}+1(2 n)_{r} B_{r-1} \ldots \ldots \ldots \ldots
\end{aligned}
$$

\section{Now assume}

$$
\begin{gathered}
\phi(z, 2 n-1)=\phi(z, 3)\left\{c_{0} \phi(z, 2 n-4)+c_{1} \phi(z, 2 n-5)+\ldots\right. \\
\left.\ldots+c_{2 n-8} \phi(z, 2)+c_{2 n-8} \phi(z, 1)+c_{2 n-4}\right\}, \\
\phi(z, 2 n)=\phi(z, 4)\left\{d_{0} \phi(z, 2 n-4)+d_{1} \phi(z, 2 n-5)+\ldots\right. \\
\left.\ldots+d_{2 n-8} \phi(z, 2)+d_{2 n-8} \phi(z, 1)+d_{2 n-4}\right\} ;
\end{gathered}
$$

it is required to find the connections between tho $a$ 's and tho c's, and between tho b's and the $d$ 's. To do this it will be sufficient to tako a special case, as the methorl is general and the result simple. As soon as we cornmence the investigation of these connections, on remombering that the even Bcinulli's numbers vanish, we find that all tho odld e's and $l$ 's vanish. 'Talsing advantago of this simplification, lid, for example,

$$
\begin{aligned}
\phi(z, 13)=\phi(z, 3)\left\{c_{0} \phi(z, 10)\right. & +c_{2} \phi(z, 8)+c_{4} \phi(z, 6)+c_{0} \phi(z, 4) \\
& \left.+c_{8} \phi(z, 2)+c_{10}\right\},
\end{aligned}
$$

then tho coeflicient of $\phi(z, 3)$ may loe explamled in powers of $z$ mul vol. $\times x$. - NO. $34 \%$. 
tabulated thus-

\begin{tabular}{|c|c|c|c|c|c|c|c|c|c|c|c|}
\hline & $z^{10}$ & $\approx 9$ & $\varepsilon^{8}$ & $2^{7}$ & $z^{n}$ & $x^{5}$ & $z^{4}$ & $z^{3}$ & $z^{2}$ & 8 & 1 \\
\hline$c_{0} \phi(z, 10)$ & $c_{11}$ & $c_{0} 10_{1} B_{0}$ & ${ }_{0} l_{0_{2}} B_{1}$ & & $c_{0} 10_{4} B_{3}$ & & $c_{0} 10_{6} B_{5}$ & & $c_{0} 10_{8} B_{7}$ & & \\
\hline$c_{2} \phi(z, 8)$ & & & $c_{2}$ & $c_{2} 8_{1} B_{0}$ & $c_{2} 8_{2} B_{1}$ & & $c_{2} 8_{4} B_{3}$ & & $c_{2} 8_{6} B_{b}$ & & \\
\hline$c_{4} \phi(z, 6)$ & & & & & $c_{4}$ & $c_{4} 6_{1} B_{0}$ & $c_{4} 6_{2} B_{1}$ & & $c_{4} 6_{4} B_{3}$ & & \\
\hline$c_{6} \phi(z, 4)$ & & & & & & & $c_{6}$ & $c_{6} 4_{1} B_{0}$ & $c_{6} 4, B_{1}$ & & \\
\hline$c_{\phi} \phi(z, 2)$ & & & & & & & & & $c_{8}$ & $c_{8} 2_{1} B_{0}$ & \\
\hline$c_{10}$ & & & & & & & & & & & $c_{10}$ \\
\hline
\end{tabular}

a function which is to be identical with the coefficient of $\phi(z, 3)$ in equation (1), when $n=7$.

$$
\begin{aligned}
& \text { Hence } \quad c_{0} 10_{1} B_{0}=a_{1} \text { or } c_{0}=-\frac{a_{1}}{5}=a_{0}=1 \text {, } \\
& c_{8} 8_{1} B_{0}=a_{3} \text { or } c_{2}=-\frac{a_{3}}{4} \text {, } \\
& c_{4} 6_{1} B_{0}=a_{3} \text { or } c_{4}=-\frac{a_{5}}{3} \text {, } \\
& c_{6} 4_{1} B_{0}=a_{7} \text { or } c_{6}=-\frac{a_{2}}{2} \text {, } \\
& c_{8} 2_{1} B_{0}=a_{9} \text { or } \quad c_{8}=-\frac{a_{9}}{1} \text {, }
\end{aligned}
$$

and

$$
c_{10}=a_{10} .
$$

In a similar manner, taking the Bernoullians of even degree, and for example, assuming

$$
\begin{gathered}
\phi(z, 14)=\phi(z, 4)\left\{d_{0} \phi(z, 10)+d_{2} \phi(z, 8)+d_{6} \phi(z, 6)+d_{0} \phi(z, 4)\right. \\
\left.+d_{8} \phi(z, 2)+d_{10}\right\},
\end{gathered}
$$

we shall find

$$
\begin{aligned}
& d_{0} 10_{1} B_{0}=b_{1} \text { or } \quad d_{0}=-\frac{b_{1}}{5}=b_{0}=1, \\
& d_{2} 8_{1} B_{0}=b_{8} \text { or } d_{2}=-\frac{b_{5}}{4}, \\
& d_{4} 6_{1} B_{0}=b_{6} \text { or } d_{4}=-\frac{b_{5}}{3},
\end{aligned}
$$




$$
\begin{aligned}
& d_{0} 4_{1} B_{0}=b_{7} \text { or } d_{0}=-\frac{b_{7}}{2}, \\
& d_{8} 2_{1} B_{0}=b_{0} \quad \text { or } \quad d_{8}=-\frac{b_{0}}{1}, \\
& d_{10}=b_{10} \text {. } \\
& \frac{\phi}{\phi} \frac{(z, 13)}{(z, 3)}=z^{10}-5 z^{0}+5 z^{8}+1 \cap z^{7}-\frac{34}{3} z^{6}-22 z^{5}+\frac{284}{21} z^{4} \\
& +\frac{219}{7} z^{3}-\frac{41}{15} z^{2}-\frac{691}{35} z-\frac{691}{105} \\
& =\phi(z, 10)-\frac{5}{2} \phi(z, 8)+\frac{22}{3} \phi(z, 6)-\frac{219}{1+4} \phi(z, 4)+\frac{691}{35} \dot{\phi}(z, 2)-\frac{691}{105} ; \\
& \frac{\phi(z, 14)}{\phi(z, 4)}=z^{10}-5 z^{0}+\frac{25}{6} z^{8}+\frac{40}{3} z^{7}-\frac{163}{15} z^{8}-\frac{526}{15} z^{5}+\frac{367}{30} z^{4} \\
& +\frac{893}{15} z^{3}+\frac{101}{15} z^{2}-\frac{691}{15} z-\frac{691}{30} \\
& =\phi(z, 10)-\frac{10}{3} \phi(z, 8)+\frac{526}{45} \phi(z, 6)-\frac{893}{30} \phi(z, 4)+\frac{691}{15} \phi(z, 2)-\frac{691}{30} \text {. }
\end{aligned}
$$

The theorem is finally stated thus, $\epsilon$ being 3 or 4 as the case may bo:

If

$\phi(z, 2 m+e)=\phi(z, \epsilon)\left\{e_{0} z^{2 m}+e_{1} z^{2 m-1}+e_{2} z^{2 m-2}+\ldots+e_{2 m-2} z^{2}+e_{2 m-1} z+e_{2 m}\right\}$, where, when $\epsilon=3$,

and when $\epsilon=4$,

$$
\begin{aligned}
e_{\mathrm{r}}=\frac{2^{r+1}-1}{2^{r}} & +\frac{2^{r}-1}{2^{r-1}}(2 m+\epsilon) B_{0}+\frac{2^{r-1}-1}{2^{r-2}}(2 m+\epsilon)_{8} B_{1} \\
& +\frac{2^{r-2}-1}{2^{r-3}}(2 m+\epsilon)_{3} B_{2}+\ldots,
\end{aligned}
$$

$e_{r}=(r+1)+r(2 m+\epsilon) B_{0}+(r-1)(2 m+\epsilon)_{2} B_{1}+(r-2)(2 m+\epsilon)_{8} B_{2}+\ldots$

then $\phi(z, 2 m+\epsilon)=\phi(z, \epsilon)\left\{\phi(z, 2 m)-\frac{e_{8}}{m-1} \phi(z, 2 m-2)\right.$

$$
\begin{aligned}
& \left.-\frac{e_{\mathrm{k}}}{m-2} \phi(z, 2 m-4)-\ldots-\frac{e_{g_{m-3}}}{2} \phi(z, 4)-\frac{e_{2 m-1}}{1} \phi(z, 2)+e_{3 m}\right\} \text {. } \\
& \text { c } 2
\end{aligned}
$$


The first 14 Bernoullians expressed in torms of lower Bernoullians are as follows :-

$$
\begin{aligned}
& \phi(z, 5)=\phi(z, 3)\left\{\phi(z, 2)-\frac{1}{3}\right\} \\
& \phi(z, 6)=\phi(z, 4)\left\{\phi(z, 2)-\frac{1}{2}\right\}, \\
& \phi(z, 7)=\phi(z, 3)\left\{\phi(z, 4)-\phi(z, 2)+\frac{1}{3}\right\}, \\
& \phi(z, 8)=\phi(z, 4)\left\{\phi(z, 4)-\frac{4}{3} \phi(z, 2)+\frac{2}{3}\right\}, \\
& \phi(z, 9)=\phi(z, 3)\left\{\phi(z, 6)-\frac{3}{2} \phi(z, 4)+\frac{9}{5} \phi(z, 2)-\frac{3}{5}\right\}, \\
& \phi(z, 10)=\phi(z, 4)\left\{\phi(z, 6)-2 \phi(z, 4)+3 \phi(z, 2)-\frac{3}{2}\right\}, \\
& \phi(z, 11)=\phi(z, 3)\left\{\phi(z, 8)-2 \phi(z, 6)+\frac{4}{4} \phi(z, 4)\right. \\
& \phi(z, 14)=\phi(z, 4)\left\{\phi(z, 10)-\frac{10}{3} \phi(z, 8)+\frac{526}{45} \phi(z, 6)-\frac{893}{30} \phi(z, 4)\right. \\
& \phi(z, 12)=\phi(z, 4)\left\{\phi(z, 8)-\frac{8}{3} \phi(z, 6)+\frac{13}{2} \cdot \phi(z, 4)\right. \\
& \phi(z, 13)=\phi(z, 3)\left\{\phi(z, 10)-\frac{5}{2}-\phi(z, 8)+\frac{22}{3} \phi(z, 6)-\frac{219}{14} \phi(z, 4)\right.
\end{aligned}
$$

Wo may note that the $a^{\prime}$ s and $b^{\prime}$ 's are connected by the relation

$$
(2 n-r) b_{r}-(2 n-r-1) b_{r-1}=2 n a_{r}-n a_{r-1}
$$


For, by obvious steps, it is easily seen that $(m=2 n)$

$$
\begin{aligned}
& b_{r}=\text { coefficient of } z^{r} \text { in } \frac{z^{r+1}-1}{z-1} \frac{d}{d z}\left\{\frac{\phi(z, m)}{z^{m-r-1}}\right\} \text {, } \\
& =\quad \quad \quad z^{m} \text { in } \frac{z^{r+1}-1}{z-1}\{m z \phi(z, m-1)-(m-r-1) \phi(z, m)\} \text {, } \\
& =\quad " \quad z^{m} \text { in } m z\left(z^{r+1}-1\right) z\left(z-\frac{1}{2}\right) \frac{\phi(z, m-1)}{\phi(z, 3)} \\
& -(m-r-1)\left(z^{r+1}-1\right) z^{2}(z-1) \frac{\phi(z, m)}{\phi(z, 4)}, \\
& =\quad " \quad z^{m} \text { in }\left(m z^{r+4}-\frac{1}{2} m z^{r+3}-m z^{8}+\frac{1}{3} m z^{2}\right) \\
& \times\left(\ldots+a_{r-1} z^{m-r-s}+a_{r} z^{m-r-1}+\ldots\right) \\
& -(m-r-1)\left(z^{r+1}-z^{r+3}-z^{3}+z^{2}\right)\left(\ldots+b_{r-1} z^{m \cdot r-3}+b_{r} z^{m-r-1}+\ldots\right),
\end{aligned}
$$

thereforo $\quad b_{r}=m a_{r}-\frac{1}{2} m a_{r-1}-(m-r-1) b_{r}+(m-r-1) b_{r-1}$,

whence the required result, viz.,

$$
(2 n-r) b_{r}-(2 n-r-1) b_{r-1}-2 n a_{r}+n a_{r-1}=0 .
$$

It is interesting to observo that this equation is trne, not on aocount of any relation among Bernoulli's numbers, but hecause the coefficient of each Burnoulli's number vanishes. Thus the torm containing $B_{p}$, in this equation is

$$
\begin{aligned}
& \left\{(2 n-r)(r-p)(2 n)_{p+1}-(2 n-r-1)(r-p-1)(2 n)_{p+1}\right. \\
& \left.-2 n \frac{2^{r-p}-1}{2^{r-p-1}}(2 n-1)_{p+1}+n \frac{2^{r-p-1}-1}{2^{r-p-2}}(2 n-1)_{p+1}\right\} B_{p},
\end{aligned}
$$

which may bo written in the form

$$
\frac{(2 n-p-1)(2 n)_{p+1}}{2^{r-p-1}} B_{p}\left(2^{r-p-1}-2^{r-p}+1+2^{r-p-1}-1\right)
$$

an expression which vanishes identically.

In fact, equation (5) is merely the arithmetical equivalent of tho differential identity

$$
\frac{d}{d z}\left\{\frac{\phi(z, 2 n)}{\phi(z, 4)}\right\}+4 \frac{\phi(z, 3)}{\phi(z, 4)} \frac{\phi(z, 2 n)}{\phi(z, 4)}=2 n \frac{\phi(z, 2 n-1)}{\phi(z, 4)} .
$$




\section{On Deep-water Waves resulting from a Limited Original \\ Disturbance. Mr. W. Burnside.}

[Read Nov. 8th, 1888.]

Cauchy," in his "Memoire sur la Théorie des Ondes," determines under certain limitations the disturbance at any point of the surface of deep water and at any time, due to an initial surface displacement over a given limited area.

Reckoning $t$ from the tim $\theta$ of the original disturbance and $x$ along the surface from its centre, his result, when the motion is in two dimensions, is that the displacement

$$
\propto \frac{t}{2^{\frac{3}{2}}} \mathrm{si}\left(\frac{g t^{9}}{4 x}+\frac{\pi}{4}\right) .
$$

The limitations mentioned are that $l / x$ and $g t^{2} l / 4 x^{9}$ should both bo very small quantities, $l$ being the length of that part of the surface in which the original displacement is seusible.

The latter limitation robs the result of most of its interest, for it will be seen later that it is not until $g t^{2} l / 4, x^{2}$ has a finite value (in the particular case first treated it is $\frac{1}{2}$ ) for a given value of $x$ that the displacement at the corresponding place reaches its maximum.

Cauchy's expression is obtrined as follows :

If the original vertical displacement at any point (the motion being supposed to start from rest) is $f(x)$, the form of the surface at any subsequent time is given by

$$
y=\frac{1}{\pi} \int_{0}^{\infty} d m \int_{-\infty}^{\infty} d a \cos m^{t} g^{t} t \cos m(a-x) f(a) .
$$

If $f(a)$ vanishes for all but very small values of $a$,

$$
\int_{-\infty}^{\infty} \cos m(a-x) f(a) d a=H \cos m x \text { approximately, }
$$

where $H$ is some constant quantity. (Canchy shews that it is here that the limitation of $g t^{3} l / 4 x^{2}$ to small values is introduced.)

Therefore $\quad y=\frac{H}{\pi} \int_{0}^{\infty} \cos m^{1} g^{\mathrm{y}} t \cos m x d \dot{m}$.

- Mem. des Sav. Etran., Vol. I., 1827. 
Next, the value of the integral

$$
\frac{1}{\pi} \int_{0}^{\infty} e^{-a m} \cos m^{\prime} g^{\prime} t \cos m x d m
$$

is calculated in the form of an infinite series, and in the result zero is written for $a$. If this is called $I$, then finally

$$
y=H I \text {. }
$$

It will be seen that, though he starts from a perfectly general form for the original surface displacement, Cauchy, as a matter of fact, introduces in the course of his work a special form, and that his result holds only for the latter under certain conditions.

Indeed his final equation may be written

$$
y=\frac{H}{\pi} L_{\bullet-0}^{i} \int_{0}^{\infty} e^{-a m} \cos m^{i} g^{i} t \cos m x d m,
$$

and hence the initial surface displacement is

$$
\begin{aligned}
y_{0} & =\frac{H}{\pi} L_{a=0}^{t} \int_{0}^{\infty} e^{-a m} \cos m x d m \\
& =\frac{H}{\pi} L_{a=0}^{t} \frac{a}{a^{2}+x^{2}} .
\end{aligned}
$$

Strictly, then, the case investigated is one in which the original displacement $\propto 1 / a^{2}+x^{2}$, and the result is obtained for such times and places that zero may be written for $a$ at a certain stage without sensible error.

In what follows I have attempted, starting first from the same initial form, but retaining the quantity a throughout, to obtain an expression for the displacement, which at a given place, shall hold through a much greater range of time than Cauchy's, and in par. ticular from which it may be possible to determine when the displacement at a given place is a maximum and also its magnitude.

Taking $c$ as the greatest initial displacement of any point of the surface, and $l$ as the length of surface over which the initial disturbance is sensible, I shall speak of a term $c(l / x)^{n}$ in the expression for the displacement at any point as of the order $(l / x)^{n}$. The displacement will be only considered for points for which $l / x$ is a small quantity.

$$
\text { Putting, then, } \quad \begin{aligned}
y_{0} & =c a \int_{0}^{\infty} e^{-a m} \cos m x d m \\
& =\frac{c a^{2}}{a^{2}+x^{2}},
\end{aligned}
$$


the initial displacement at any distant point is of the order $(l / x)^{2}$, and this is to be considered vanishingly small.

The theory of deep-water waves shews that, if the surface be initially displaced but motionless, the typical solution is

$$
y=A \cos m x \cos m^{\prime} g^{\prime} t,
$$

the correspouding initial form being

$$
y=A \cos m x ;
$$

and by an application of Fourier's theorem, uny arbitrary furm of free surface may be built up from this typical solution.

Hence at once the form of the surface at time $t$, corresponding to the above value $y_{0}$, for the initial displacement, is

$$
y=c a \int_{0}^{\infty} e^{-a m-7 s} m x \cos m^{\prime} g^{\dagger} t d m
$$

By expanding the term $\cos m^{\prime} g^{\prime} t$, and integrating term by term, this becomes

$$
y=\frac{c a}{\left(a^{2}+x^{2}\right)^{1}} \sum_{0}^{\infty} \frac{(-1)^{n} n !}{2 n !} \frac{\left(g t^{2}\right)^{n}}{\left(a^{2}+x^{2}\right)^{1 n}} \cos \left(\overline{n+1} \tan ^{-1} \frac{x}{a}\right)
$$

or, if

$$
\frac{g t^{2}}{\left(a^{3}+x^{3}\right)^{4}}=2 x, \quad \tan ^{-1} \frac{x}{a}=\beta,
$$

$$
y=c \cos \beta\left[\cos \beta-z \cos 2 \beta+\frac{z^{2}}{1.3} \cos 3 \beta-\frac{z^{\circ}}{1.3 .5} \cos 4 \beta+\ldots\right] \text {. }
$$

Now, it may bo easily verified, by integrating by parts, that

$$
\begin{aligned}
& 2 \cos \beta[z \cos 2 \beta-\left.\frac{z^{2}}{1.3} \cos 3 \beta+\frac{z^{3}}{1.3 .5} \cos 4 \beta-\ldots\right] \\
&=(z \cos \beta)^{3} \int_{0}^{z \cos \beta} \frac{e^{-y / 2} \cos \left(2 \beta-\frac{1}{2} y \tan \beta\right)}{(z \cos \beta-y)^{1}} d y,
\end{aligned}
$$

and honco

$$
y=c \cos ^{2} \beta-\frac{1}{2} c(z \cos \beta)^{1} \int_{0}^{s \cos \beta} \frac{\theta^{-y / 2} \cos \left(2 \beta-\frac{1}{2} ! ! \tan \beta\right)}{(z \cos \beta-y)^{1}} d y .
$$

If in the integral the variable be changed by the substitation

$$
y=z \cos \beta-2 y^{\prime} \cot \beta,
$$


1888.] resulting from a Limited Original Disturbance.

we have, after reduction,

$$
\begin{gathered}
y=c \cos ^{\prime} \beta-c \cos \beta\left(\frac{z}{2 \sin \beta}\right)^{\prime} e^{-i n \cos \rho} \\
\times\left[\cos \left(2 \beta-\frac{z \sin \beta}{2}\right) \int_{0}^{1 \sin \beta} \frac{e^{y^{\prime} \cot \beta} \cos y^{\prime}}{y^{\prime}} d y^{\prime}\right. \\
\left.-\sin \left(2 \beta-\frac{z \sin \beta}{2}\right) \int_{0}^{1+\sin \rho} \frac{e^{y^{\prime} \cot \beta} \sin y^{\prime}}{y^{\prime}} d y^{\prime}\right] .
\end{gathered}
$$

The integrals now to be evaluated are of the form

$$
\int_{0}^{p} \frac{e^{y x} \cos x}{x^{1}} d x
$$

where $q$ is a very small numerical quantity. By expanding the exponential and integrating each term by parts, the following expressious are at once obtained for the two integrals :-

$$
\begin{aligned}
& \int_{0}^{p} \frac{e^{q x} \cos x}{x^{4}} d x=\left(1-\frac{1.3}{2.4} q^{2}+\frac{1.3 .5 .7}{2.4 .6 .8} q^{4}-\ldots\right) \int_{0}^{p} \frac{\cos x}{x^{1}} d x \\
& +\left(-\frac{1}{2} q+\frac{1.3 .5}{2 \cdot 4 \cdot 6} q^{s}-\frac{1.3 .5 \cdot 7 \cdot 9}{2 \cdot 4 \cdot 6 \cdot 8 \cdot 10} q^{s}+\ldots\right) \int_{0}^{p} \frac{\sin x}{x^{3}} d x \\
& +\frac{\sin p}{p^{4}}\left(p q+\frac{\eta^{2} q^{2}}{2 !}+\frac{p^{8} q^{8}}{3 !}+\ldots\right) \\
& +\frac{\cos p}{p^{\frac{3}{5}}}\left(\frac{3}{2} \frac{p^{3} q^{2}}{2 !}+\frac{5}{2} \frac{p^{9} q^{8}}{3 !}+\frac{7}{2} \frac{p^{4} q^{4}}{4 !}+\ldots\right) \\
& -\frac{\sin p}{p^{\frac{8}{5}}}\left(\frac{3.5}{2.2} \frac{p^{8} q^{8}}{3 !}+\frac{5.7}{2.2} \frac{p^{4} q^{4}}{4 !}+\ldots\right) \\
& -\frac{\cos p}{p^{\frac{1}{3}}}\left(\frac{3.5 .7}{2.2 .2} \frac{p^{4} q^{4}}{4 !}+\frac{5.7 .9}{2.2 .2} \frac{p^{5} q^{6}}{5 !}+\ldots\right)+\text { ctc. } \\
& \int_{0}^{p} \frac{e^{q x} \sin x}{x^{3}} d x=\left(1-\frac{1.3}{2.4} q^{2}+\frac{1.3 .5 .7}{2.4 \cdot 6.8} q^{4}-\ldots\right) \int_{0}^{p} \frac{\sin x}{x^{1}} d x \\
& +\left(\frac{1}{2} q-\frac{1.3 .5}{2.4 \cdot 6} q^{9}+\ldots\right) \int_{0}^{p} \frac{\cos x}{x^{4}} d x \\
& -\frac{\cos p}{p^{1}}\left(p q+\frac{p^{2} q^{2}}{2 !}+\ldots\right) \\
& +\frac{\sin p}{p^{3}}\left(\frac{3}{2} \frac{p^{2} q^{2}}{2 !}+\frac{5}{2} \frac{p^{3} q^{8}}{3 !}+\ldots\right) \\
& +\frac{\cos p}{p^{3}}\left(\frac{3.5}{2.2} \frac{p^{3} q^{3}}{3 !}+\frac{5.7}{2.2} \frac{p^{4} q^{4}}{4 !}+\ldots\right) \\
& -\frac{\sin p}{p^{!}}\left(\frac{3.5 .7}{2.2 .2} \frac{\mu^{4} q^{4}}{1 !}+\frac{5.7 .9}{2.2 .2} \frac{p^{5} q^{5}}{5 !}+\ldots\right)-\text { ctc. }
\end{aligned}
$$


The general term of the.part occurring after the first two lines in either of these integrals is

$$
\pm \frac{\cos p}{p^{1(2 n-1)}}\left[\frac{2 n-1.2 n-3 \ldots 3}{2^{n-1}} \frac{(p q)^{n}}{n !}+\frac{\overline{2 n+1} \ldots 5}{2^{n-1}} \frac{(p q)^{n+1}}{n+1 !}+\ldots\right],
$$

and the quantity in brackets is clearly less than

$$
(p q)^{n-1}\left(e^{p q}-1\right) \text {. }
$$

Tho general term of the series is therefore in magnitude less than

$$
\frac{\sin p}{p^{t}}\left(e^{p q}-1\right) q^{n-1} \text {. }
$$

Hence, $q$ being very small, we.have as first approximations

$$
\begin{aligned}
& \int_{0}^{p} \frac{e^{q x} \cos x}{x^{l}} d x=\int_{0}^{p} \frac{\cos x}{x^{4}} d x+\frac{\sin p}{p^{l}}\left(e^{p q}-1\right), \\
& \int_{0}^{p} \frac{e^{q x} \sin x}{x^{l}} d x=\int_{0}^{p} \frac{\sin x}{x^{l}} d x-\frac{\cos p}{p^{l}}\left(e^{p q}-1\right),
\end{aligned}
$$

the next terms of either part of either integral being of the order $q$ times those written.

Also $\left(e^{m q}-1\right) / p^{\prime}$ increases continually with $p$, and for moderate values of $p q$ (which will be found to correspond to the case here to be considered) is of the order $q^{\prime}$, and therefore in the expression of each integral the second terms written are intermediate in magnitude between the first and those omitted.

Finally, as is well known,

$$
\begin{aligned}
& \int_{0}^{p} \frac{\cos x}{x^{t}} d x=\sqrt{\frac{\pi}{2}}+\frac{\sin p}{p^{1}}-\frac{1}{2} \frac{\cos p}{p^{!}}-\frac{1.3}{2.2} \frac{\sin p}{p^{3}}+\ldots \\
& \int_{0}^{1} \frac{\sin x}{x^{l}} d x=\sqrt{\frac{\pi}{2}}-\frac{\cos p}{p^{3}}-\frac{1}{2} \frac{\sin p}{p^{\frac{1}{3}}}+\frac{1.3}{2.2} \frac{\cos p}{p^{t}}+\ldots
\end{aligned}
$$

where the divergent series may be used as approximations by stopping at or before the smallest terms.

Before substituting in the expression for the displacement the valnes of the integrals now found, it will be well to consider the magnitudes of some of the quantities involved, with a view to ncglecting systematically terms $q$ times those retained.

Thus $\quad \cos \left(2 \beta-\frac{z \sin \beta}{2}\right)$ and $\sin \left(2 \beta-\frac{z \sin \beta}{2}\right)$ 
differ from

$$
-\cos \left(\frac{z \sin \beta}{2}\right) \text { and } \sin \left(\frac{z \sin \beta}{2}\right) \text {, }
$$

by quantities of the order $q$ times these latter quantities; also

$$
p=\frac{z \sin \beta}{2}=\frac{g t^{2} x}{4\left(x^{2}+a^{2}\right)},
$$

and hence, except in the circular functions $g t^{2} / 4 x$, may certainly be written for $p$; so also

$$
\frac{z \cos \beta}{2}(=p q)=\frac{g t^{2} u}{4 x^{2}}, \quad \cos \beta=\frac{a}{x},
$$

and

$$
\frac{z}{2 \sin \beta}=\frac{g t^{2}}{4 x} \text { approximately. }
$$

Hence, retaining $p$ for $\frac{1}{2} z \sin \beta$ in the circular functions, wo get

$$
\begin{aligned}
& y=\frac{c \alpha}{x}\left(\frac{g t^{2}}{4 x}\right)^{2} e^{-0 t^{2} \alpha / 4 x^{2}} \\
& \times\left[\cos p\left\{\sqrt{\frac{\pi}{2}}+\left(\frac{g t^{2}}{4 x}\right)^{-1} e^{g t^{2} 0 / 4 x^{2}} \sin p-\frac{1}{2}\left(\frac{g t^{2}}{4 x}\right)^{-1} \cos p-e t c .\right\}\right. \\
& \left.+\sin p\left\{\sqrt{\frac{\pi}{2}}-\left(\frac{g t^{2}}{4 x}\right)^{-t} e^{\rho t^{2} 0 / 4 x^{2}} \cos p-\frac{1}{2}\left(\frac{g t^{2}}{4 x}\right)^{-1} \sin p+\text { etc. }\right\}\right] \\
& =\frac{c a}{x}\left(\frac{q t^{2}}{4 x}\right)^{2} e^{-o t^{2} \alpha / x^{2}} \\
& \times\left[\sqrt{ } \pi \sin \left(p+\frac{\pi}{4}\right)-\frac{1}{2}\left(\frac{g t^{2}}{4 x}\right)^{-1}+\frac{1.3 .5}{2.2 .2}\left(\frac{g t^{2}}{4 x}\right)^{-1}-\ldots\right] .
\end{aligned}
$$

With the same coefficient outside the bracket, the most important of the omitted periodic terms inside is

$$
\frac{3 \sqrt{ } \pi}{2} \frac{a}{x} \cos \left(p+\frac{\pi}{4}\right)
$$

The ultimately divergent series following the first term inside the bracket, may, as in the case of the two from which it is derived, be used as an approximation by stopping before the smallest tern ; and, as it converges for three terms if $g t^{2} / 4 x$ is greater than 4 , the above expression will servo for the calculation of $y$ at a given point for any values of the time not less than $4 \sqrt{\mathrm{x} / \mathrm{g}}$.

For small values of $t$ the original series is oertainly the best form from which to calculate $y$; and from it may be seen that for such values $y$ is of tho order $(a / x)^{2}$, as it is initially. Tho expression for $y$ now found shows that, whon $g t^{2} / 4 x$ is a moderately largo number, $y$ is of tho 
order $a / x$; but also indicates that, when $g t^{2} / 4 x$ is comparable with $x / a, y$ is of the order $\sqrt{a / x}$. For values of $t$ such as this all terms following $\sqrt{ } \pi \sin (p+\pi / 4)$ may certainly be omitted as smaller than terms already neglected. Moreover, when $g t^{2} / 4 x$ is comparable with $x^{2} / a^{2}$, the valuo of $y$ will, owing to the factor $e^{-g t^{2}} / / x^{*}$, be again extremely small, and hence in the term $\sin (p+\pi / 4)$ we may safely writo $g t^{2} / 4 x$ for $p$. We have finally then, as an expression for the displacement at a given place, as long as its magnitude is comparable with its maximum value,

$$
y=\sqrt{ } \pi \frac{c a}{x}\left(\frac{g t^{2}}{4 x}\right)^{d} e^{-g t^{2} \alpha / 4 x^{2}} \sin \left(\frac{g t^{3}}{4 x}+\frac{\pi}{4}\right) .
$$

The greatest value of

$$
\left(\frac{g t^{2}}{4 x}\right)^{1} e^{-g t^{3} / / x^{2}}
$$

for a particular value of $x$, is given by

$$
g t^{2} a=2 x^{2},
$$

and the ratio of the logarithmic differential coefficient of $\left(\frac{g t^{2}}{4 x}\right)^{1} e^{-g t^{2} / \omega^{2}}$ to that of $g t^{3} / 4 x$ is

$$
1-\frac{g t^{2} a}{2 x^{2}}
$$

hence, when, for a given value of $x, y$ is near its greatest value, $\sin \left(\frac{g t^{2}}{4 x}+\frac{\pi}{4}\right)$ changes very rapidly compared to its coefficient; or, in other words, to determine the maximum value of $y$, the coefficient only need bo considered.

The greatest amplitude of displacement at the point $x$ is therefore

$$
\sqrt{\frac{\pi}{2}} c e^{-1}\left(\frac{a}{x}\right)
$$

and the amplitude has this value when, as seen above,

$$
t=x \sqrt{\frac{2}{g a}} .
$$

$\Delta$ lso, if $g t^{3} / 4 x$ increases by $2 \pi$ between times $t$ and $t^{\prime}$, then

$$
t^{\prime}-t=\frac{\delta \pi x}{g\left(t^{\prime}+t\right)}
$$

or, in words, the poriod of the wave motion at $x$, when the amplitude is grcatest, is

$$
2 \pi \sqrt{\frac{2 a}{y}}
$$

and the length of the waves is $+1 \pi$ a. 
For places at which the displacement has not reached its maximum the period and wave-length are greater, and for those for which tho greatest displacement is past they aro less than those quantities.

The energy of the initial displncement varies as $c^{2} a$, and the extent of surface over which it is sensible varies as a. The above results may then be expressed as follows.

For the same form of initial displacement-

(i.) The greatest amplitude of displacement at any point varies as the square root of the whole energy of the motion, and inversely as the square root of the distance from the originally disturbed area.

(ii.) The greatest disturbance is propagated with a uniform velocity, varying as the square root of the space over which the original dis. turbance is sensible; and

(iii.) The wave-length of the motion, when greatest, varies as the space ovor which the original disturbance is sensible.

These results are proved only for the case now considered; but, from the nature of the problem, thore can be little doubt but that similar statoments may be made with regard to the motions resulting from any other form of initial displacement. Indeed this is shown to bo the caso later for two other forms.

To represent the resilts in a complete way graphically would involve very considerable labour; but, omitting the periodic term in the expression for the displacement, the magnitude of the motion only may bo represented as follows.

If in the general expression for $y$ the periodic factor be omitted, then, when $x$ is recrarded as constant,

$$
y=A t e^{-\cdot t^{2},}
$$

and, when $t$ is regarded as constant,

$$
y=\frac{B}{x !} e^{-s / x^{3}},
$$

where $A, B, \alpha, \beta$ are constant quantities. The general forms of the curves with these equations will not depend on the ralues of tho constants, which affect only the relative scales parallel to the two axes ou which they are drawn.

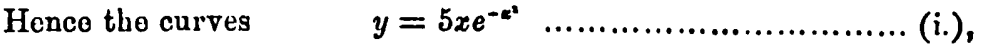

$$
\begin{aligned}
& y=\frac{5}{x^{i}} e^{-i / x^{*}} \ldots \ldots \ldots \ldots \ldots \ldots \ldots \ldots \text { (ii.), }
\end{aligned}
$$

(where the numerical factor is introduced to give suitable propor- 
tions to the figures,) will show graphically-the first, how the amplitude of the displacement varies with the time at a particular place; the second, how the amplitudes vary from place to place at a given time.

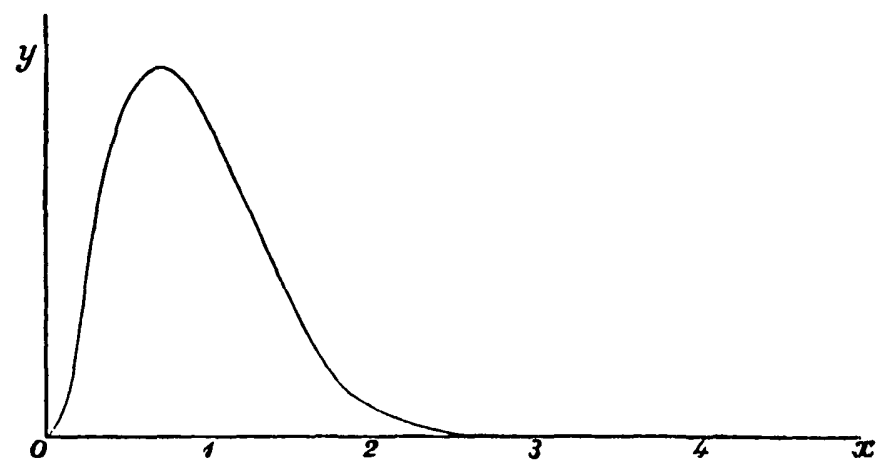

Fio. 1.

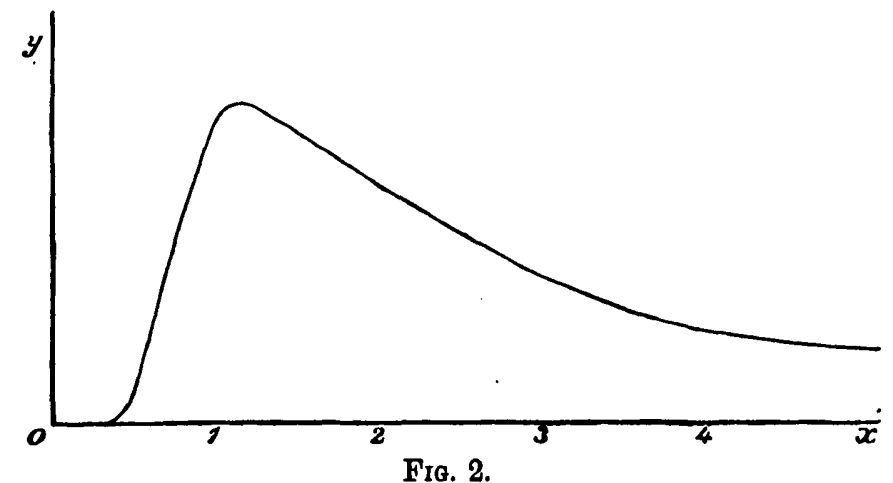

These curves enable us to further interpret the formula for $y$ in a general way as follows.

At a particular point the disturbance dies away from its maximum value at a slower rate than it grows up to it ; though, the first curve not being very unsymmetrical on either side of its maximum ordinate, the rise and dying away of the disturbance at a particular point will not be very dissimilar.

At a particalar time, the greater part of the surface sensibly disturbed will be beyond the point of maximum disturbance, on the near side of which the amplitude rapidly diminishes to an insensible amount.

These resalts, which at first sight appear almost to contradict 
each other, are reconciled at once when it is noticed that at any instant the extont of sensibly disturbed surface (i.e., the amount of surface in which the amplitude of the disturbance is not less than a givon sub-multiple of the existing greatest disturlanco) increases in direct proportion with the distance of the point of greatest disturbance from the origin.

It appoars certain that, for the particular form of original disturbance hitherto considered, $y$ cannot be exactly expressed in terms of $x$ and $t$ otherwise than by an infinito scries. It is not, howover, difficult artificially to build up forms for the original disturbanco, such that the value of $y$ for any values of $x$ and $t$ can bo expressed in finite terms; and if these are chosen so that tho extent of surfaco over which the original disturbanco is sonsiblo is limited, the results will serve as a sort of test of tho accuracy of tho approximato value of $y$ obtained above, and of the law of the motion deduced from it.

In particular, if

$$
y_{0}=A \int_{0}^{\infty} m^{i+1} \theta^{-a m} \cos m x d m,
$$

where $i$ is a positive integer, the resulting value of $y$ may be expressed in finito torms.

Taking the case of $i$ zero, put

$$
\begin{aligned}
y_{0} & =\frac{2 c}{\sqrt{ } \pi} \cdot a^{t} \int_{0}^{\infty} m^{4} e^{-a m} \cos m x d m \\
& =c\left(\frac{a^{2}}{a^{2}+x^{2}}\right)^{2} \cos \left(\frac{3}{2} \tan ^{-1} \cdot \frac{x}{a}\right) .
\end{aligned}
$$

Then

$$
y=\frac{2 c u^{\pi}}{\sqrt{\pi}} \int_{0}^{\infty} m^{t} e^{-a m} \cos m x \cos m^{t} g^{4} t d m
$$

Put

$$
\begin{aligned}
m x & =m^{\prime 2}, \\
\tan ^{-1} \frac{x}{a} & =\beta, \\
\frac{g t^{2}}{4: c} & =p,
\end{aligned}
$$

and therefore

$$
y=\frac{4 c}{\sqrt{\pi}}\left(\begin{array}{c}
a \\
: c
\end{array}\right)^{3} \int_{0}^{\infty} m^{3} e^{-m^{2} \cot \theta} \cos n^{2} \cos 2 m y^{1} d m
$$


This integral mny he derlnced from the known form

$$
\int_{0}^{\infty} e^{-q m^{2}} \cos 2 m p^{l} d n=\frac{\sqrt{ } \pi}{2} \frac{1}{q^{l}} e^{-p / q} .
$$

Differentiato this with respect to $q$, giving

$$
\int_{0}^{\infty} m^{2} e^{-q^{2}} \cos 2 m p^{4} d m=\frac{\sqrt{ } \pi}{4}\left[\frac{1}{q^{4}}-\frac{2 p}{q^{i}}\right] e^{-p / q} .
$$

Now, for $q$ write $\cot \beta+i$, and equate real parts; then

$$
\begin{aligned}
& \int_{0}^{\infty} m^{2} e^{-m^{2} \cot \beta} \cos m^{2} \cos 2 m p^{1} d m \\
& =\operatorname{real} \text { part of } \frac{\sqrt{ } \pi}{4}\left[\sin ^{i} \beta e^{-3 i \beta / 2}-2 p \sin ^{i} \beta e^{-6 i s / 2}\right] e^{-p \sin \beta[\cos \beta-i \sin \rho \mid} \\
& =\frac{\sqrt{ } \pi}{4} \sin 3 \beta e^{-p \sin g \cos \beta}\left[\cos \left(p \sin ^{2} \beta-\frac{3 / 3}{2}\right)\right. \\
& \left.-2 p \sin \beta \cos \left(p \sin ^{2} \beta-\frac{5 \beta}{2}\right)\right] \text {. }
\end{aligned}
$$

[Tho sulustitution of a complex value for $q$ may bi justified as follows :- 'ho integral

$$
\int_{0}^{\infty} m m^{2} e^{-q m^{2}} \cos 2 m p^{4} d m
$$

is the sum of a scries of integrals of the form

$$
\int_{0}^{\infty} m^{2 n} e^{-q m^{2}} d m
$$

The result of writing $\cot \beta+i$ for $q$ is the same, neglecting a factor in the integrand independent of $m$, as that of writing

$$
\left(\cos \frac{\beta}{2}+i \sin \frac{\beta}{2}\right) m^{\prime} \text { for } m \sqrt{q \sin \beta},
$$

and since $\beta / 2$ is necessarily less than $\pi / 4$, the known theory shows at once that the result of the substitution is correct.]

Finally, then, neglecting terms of the order a/x multiplied by those retained; so as to compare this result directly with the former one,

$$
y=c\left(\frac{a}{x}\right)^{\prime} e^{-0 t^{2} \alpha / s x^{2}}\left[\frac{g t^{2}}{2, i} \sin \left(\frac{g t^{2}}{4 \cdot x}+\frac{\pi}{4}\right)-\cos \left(\frac{g t^{2}}{4 x}+\frac{\pi}{4}\right)\right],
$$

and for values of $y$ at auy place comparahle with its mnximum value, the second ter'm inside the bracket may be neglected in comparison with the first. 
It will be clear, on inspection of the value of $y$ just obtained, that, with the new form of initial disturbance now considered, the general laws of the motion are the same as those already given. When, however, the particular results for tho two given forms are compared, it will be seen that the initial form scems to have a decided effect on the resulting motion.

\begin{tabular}{|c|c|c|}
\hline Initial ordinate ......... & $\frac{c u^{2}}{\alpha^{2}+x^{2}}$ & ce $\left(\frac{u^{2}}{a^{2}+x^{2}}\right)^{2} \cos \left(\frac{3}{2} \tan ^{-1} \frac{x}{a}\right)$ \\
\hline $\left.\begin{array}{l}\text { Whole energy of } \\
\text { motion }\end{array}\right\} \ldots$ & $\frac{\pi}{4} g c^{2} a$ & $\frac{1}{2} g c^{2} a$ \\
\hline 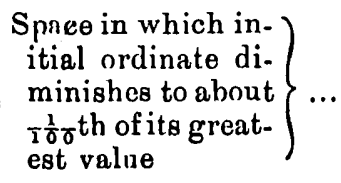 & $10 a$ & $21 a$ \\
\hline $\left.\begin{array}{l}\text { Maximum ampli- } \\
\text { tude at place } x\end{array}\right\} \ldots$ & $\sqrt{\frac{\pi}{2}} c \theta^{-1}\left(\frac{\alpha}{x}\right)^{\prime}$ & $2 c e^{-1}\left(\frac{\alpha}{x}\right)^{\prime}$ \\
\hline $\left.\begin{array}{r}\text { Rate of propnga- } \\
\text { tion of greatest } \\
\text { disturbance }\end{array}\right\} \ldots$ & $\sqrt{g u / 2}$ & $\sqrt{y u / 4}$ \\
\hline $\left.\begin{array}{c}\text { Wave-lingth of } \\
\text { motion when am- } \\
\text { plitude is greatcst }\end{array}\right\} .$. & $4 \pi a$ & $2 \pi a$ \\
\hline
\end{tabular}

The two cases hitherto considered are alike in this, that, the initial displacement being continuous, the extent of surface over which they are sensible can only be assigned by a convention as to what small quantities are to he considered negligible. It can hardly be doubted bat that such a plienomenon as the Krakatao waves would be represented more nearly in symbols by a discontinuous initial form, the ordinate of which is strictly zero beyond a cortain point. It is in. teresting, tro, to notice how far the analytical expression for the displacement in snch a case differs from those already considered. Suppose then that, for valnes of $x \cdot$ between $+a$ and $-a$, the initial displacement is $c$, and that for all other values of $x$ it is zero.

$$
\begin{aligned}
& \text { Then } \quad y_{0}=\frac{c}{\pi} \int_{0}^{\infty} \frac{\sin (x+a) m-\sin (x-a) m}{m} d i m, \\
& \text { and } \quad y=\frac{c}{\pi} \int_{0}^{\infty} \frac{\sin (t+a) m-\sin (x-a) m}{m} \cos m i d t d m .
\end{aligned}
$$

vor. XX.-No. 34.5. 
The integral to whose value it is necessary to approximate in this case is

$$
\int_{0}^{\infty} \frac{\sin m \cos m \cdot p^{i}}{m} d m
$$

whero

$$
p=\frac{g t^{3}}{x \pm a}
$$

and it may be readily expressed in a series of ascending powers of $p$ from which, for moderate values of $p$, tho displacement may be calcuJated.

Thus, if $f(p)$ denote the integral,

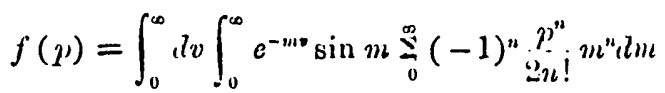

$$
\begin{aligned}
& =\frac{1}{\Sigma_{i}} \int_{0}^{\infty} d l v \coprod_{0}^{\infty}(-1)^{n} \frac{n !}{2 n !} p^{n}\left(\frac{1}{(v-i)^{n+1}}-\frac{1}{(v+i)^{n+1}}\right) \\
& =\frac{\pi}{2}+\sum_{1}^{\infty}(-1)^{n+1} \frac{2 n !}{4 n+2 !} \mu^{2 n+1}, \\
& \text { and } \quad y=\frac{c}{\pi}-\left[f\left(\frac{g t^{4}}{i+a}\right)-f\left(\frac{g t^{3}}{2-a}\right)\right] \text {. }
\end{aligned}
$$

As long as $y t^{\prime \prime} / x$ is small compared with $x / a$ tho abovo value of $y$ is clearly of the order a/:c, and as wo may now anticipate that tho greatest displiacoment al any point is of the order $(a / a)$, it is neecssary to consider the value of $f(p)$ for largo values of $p$ for which purpose the serics is of little use.

Writing $v^{2}$ for $m p$ in tho integral, we have

$$
\begin{aligned}
f(p) & =2 \int_{0}^{\infty} \frac{\sin v^{4} / p \cos v}{v} d v \\
& =2\left[\frac{\sin v^{2} / p \sin v}{v}\right]_{0}^{\infty}-\frac{4}{p} \int_{0}^{\infty} \cos v^{2} / p \sin v d v+2 \int_{0}^{\infty} \frac{\sin v^{2} / p \sin v}{v^{2}} d v .
\end{aligned}
$$

The integrated term vanishes at tho limits, and the last term

$$
=2 \int_{n}^{\infty} \frac{d p}{l^{2}} \int_{0}^{\infty} \cos r^{2} / p \sin n d u
$$

therefore $\quad f(p)=\left[-\frac{4}{p}+2 \int_{1}^{\infty} \frac{d p}{p^{2}}\right] \int_{0}^{\infty} \cos v^{*} / p \sin v d v$. 
1888.] resulting from a Limited Original Disturbance.

$$
\begin{aligned}
& \text { Again, } \int_{0}^{\infty} \cos v^{2} / p \sin v d v=\frac{1}{2} \int_{0}^{\infty} \sin \left[\left(\frac{v}{p^{4}}+\frac{p^{1}}{2}\right)^{2}-\frac{p}{4}\right] d v \\
& -\frac{1}{2} \int_{0}^{\infty} \sin \left[\left(\frac{v}{p^{4}}-\frac{p^{4}}{2}\right)^{2}-\frac{p}{4}\right] d v \\
& =\frac{1}{2} p^{4} \cos \frac{p}{4}\left[\int_{\frac{1}{2} p^{4}}^{\infty} \sin u^{3} d u-\int_{-\frac{1}{2} p^{4}}^{\infty} \sin u^{y} d u\right] \\
& -\frac{1}{2} p^{4} \sin \frac{p}{4}\left[\int_{\frac{1}{2} u^{4}}^{\infty} \cos u^{2} d u-\int_{-\frac{1}{2} p t}^{\infty} \cos u^{2} d u\right] \\
& =\frac{1}{2} p^{\frac{1}{2}} \cos \frac{p}{4}\left[-\sqrt{\frac{\pi}{2}}+2 \int_{\frac{1}{2} \eta^{2}}^{\infty} \sin u^{2} d u\right] \\
& -\frac{1}{2} p^{4} \sin \frac{p}{4}\left[-\sqrt{ } \cdot \frac{\pi}{2}+2 \int_{\frac{1}{2} p}^{\infty} \cos u^{2} d u\right]
\end{aligned}
$$

and, using the values of the integrals already given in a slightly different form,

$$
\begin{aligned}
& =\frac{1}{2} p^{\frac{1}{2}} \cos \frac{p}{4}\left[-\sqrt{\frac{\pi}{2}}+\frac{\cos p / 4}{(p / 4)^{\frac{1}{3}}}+\frac{1}{2} \frac{\sin p / 4}{(p / 4)^{\frac{3}{3}}}-\frac{1 \cdot 3}{2 \cdot 2} \frac{\cos p / 4}{(p / 4)^{\frac{1}{3}}}-\ldots\right] \\
& -\frac{1}{2} p^{2} \sin \frac{p}{4}\left[-\sqrt{\frac{\pi}{2}}-\frac{\sin p / 4}{(p / 4)^{\frac{1}{2}}}+\frac{1}{2} \frac{\cos p / 4}{(\nu / 4)^{\frac{1}{2}}}+\frac{1.3}{2.2} \frac{\sin p / 4}{(p / 4)^{\frac{1}{2}}}-\ldots\right] \\
& =-\frac{\sqrt{ } \pi}{2} \mu^{4} \cos \left(\frac{p}{4}+\frac{\pi}{4}\right)+1-\frac{1 \cdot 3}{2 \cdot 2}\left(\frac{4}{p}\right)^{2}+\ldots,
\end{aligned}
$$

where, as beforc, the divergent series may be used as an approximation by stopping beforo the smallest term.

Tho leading part of

$$
\int_{n}^{\infty} \frac{d p}{p^{2}} \int_{0}^{\infty} \cos v^{2} / p \sin v d v
$$

when $p$ is great, will be

$$
-\frac{1}{2} \sqrt{ } \pi \int_{p}^{\infty} \frac{\cos (p+\pi) / 4}{p^{3}} d p+\int_{p}^{\infty} \frac{d p}{p^{2}}
$$

or

$$
\frac{1}{p}+\text { terms of order } \frac{1}{p^{3}} \text {, etc.; }
$$

lience $f(p)=2 \sqrt{ } \pi \frac{1}{p^{4}} \cos \frac{p+\pi}{4}-\frac{2}{p}+$ terms of order $\frac{1}{p^{\frac{3}{3}}}$, etc.

$$
\text { เ } 2
$$


The resulting value of the displacement is

$$
\begin{aligned}
y= & \frac{2 c}{\sqrt{ } \pi}\left[\left(\frac{x+a}{g t^{2}}\right)^{t} \cos \left(\frac{g t^{2}}{4(x+a)}+\frac{\pi}{4}\right)-\left(\frac{x-a}{g t^{2}}\right)^{\prime} \cos \left(\frac{g t^{2}}{4(x-a)}+\frac{\pi}{4}\right)\right] \\
& -\frac{2 c}{\pi}\left[\frac{x+a}{g t^{2}}-\frac{x-a}{g t^{2}}\right] \\
& + \text { etc. } ;
\end{aligned}
$$

and when, as before, ternıs of the order $a / x$ multiplied into those re. tained are neglected,

$$
\begin{aligned}
y=\frac{2 c}{\sqrt{ } \pi}\left(\frac{x}{g t^{4}}\right)^{\prime} & {\left[\cos \left(\frac{g t^{2}}{4(x+a)}+\frac{\pi}{4}\right)-\cos \left(\frac{g t^{2}}{4(x-a)}+\frac{\pi}{4}\right)\right.} \\
+ & \left.\frac{a}{2 x}\left\{\cos \left(\frac{g t^{2}}{4(x+a)}+\frac{\pi}{4}\right)+\cos \left(\frac{g t^{2}}{4(x-a)}+\frac{\pi}{4}\right)\right\}\right] .
\end{aligned}
$$

So long as $g t^{2} / x$, though considerable, is small compared with $x / a$, the two pairs of terms in the bracket are both of the order $a / x$. When, however, $g t^{2} /: e$ and $x / a$ aro of the same order of magnitude, the second pair of terms are of the order $a / x$ compared with the first. Neglecting, therefore, the last pair, as continually before, and for reasons already given neglecting terms of the order $g t^{2} a^{2} / x^{8}$ in the arguments of the periodic terms, we have, finally,

$$
y=\frac{4 c}{\sqrt{ } \pi}\left(\frac{x}{g t^{2}}\right)^{4} \sin \frac{g t^{2} a}{4 x^{2}} \sin \left(\frac{g t^{2}}{4 x}+\frac{\pi}{4}\right) .
$$

Here, again, $\sin \left(\frac{g t^{2}}{4 t}+\frac{\pi}{4}\right)$ changes rapidly as compared with

$$
\left(\frac{x}{g t^{2}}\right)^{\prime} \sin \frac{g t^{2} a}{4 x^{2}}
$$

and to determine the magnitude of the disturbance at any time the latter quantity need alone be considered. Its maximum valucs are given by

$$
0=\frac{1}{t^{2}} \sin \frac{g t^{3} a}{4 x^{2}}-\frac{g a}{2 x^{3}} \cos \frac{g t^{2} a}{4 x^{2}}
$$

or

$$
\tan \theta=2 \theta \text {, }
$$

where

$$
\theta=\frac{g t^{2} a}{4 x^{2}} \text {. }
$$

The roots of this eqnation, omitting zero, form an ascending series 
$\theta_{1}, \theta_{2}$, eto., such that $\theta_{n}$ approximates rapidly to $(2 n-1) \frac{1}{8} \pi$ as $n$ inoncases. The first two roots are approximately

$$
\begin{aligned}
& \theta_{1}=1 \cdot 165, \\
& \theta_{2}=4 \cdot 622 .
\end{aligned}
$$

To each root of the equation corresponds a maximum value of the amplitude for the particular value of $x$ considered, that. corresponding to $\theta_{n}$ being

$$
\frac{2 c}{\sqrt{ } \pi}\left(\frac{a}{x}\right)^{\prime} \frac{\sin \theta_{n}}{\theta_{n}^{4}}
$$

Now

$$
\frac{\sin \theta_{1}}{\theta_{1}^{2}}=851
$$

and

$$
\frac{\sin \theta_{3}}{\theta_{2}^{t}}=-\cdot 463
$$

and

$$
\frac{\sin \theta_{n}}{\theta_{n}^{\frac{1}{2}}}=(-1)^{n-1} \sqrt{\frac{2}{(2 n-1) \pi}} \text {, nearly, }
$$

so that the first of these successive maxima is the greatest in absolute magnitude, and they diminish continually.

The maximum corresponding to each root of the equation

$$
\tan \theta-2 \theta=0
$$

is propagated with uniform velocity, and the magnitude of each maximum amplitude is, as before, directly proportional to the square root of the whole energy, and inversely as the square root of the distance from the original disturbance.

The intervals between successive maxima are periods of time comparable with that elapsing between the original disturbance and the first maximurn.

A second feature distinguishing this case from those before, is in the much greater duration, ceteris paribus, of the sensible disturbance at a particular point.

If $t_{0}$ is the time elapsing between the original disturbance and the chief maximum at the point considered, $t_{0} / \log n$ is an approximato measure of the time that elapses in the former case before the amplitude diminishes to $1 / n^{\text {th }}$ of its greatest value; while in the case now under consideration $(n-1) t_{0}$ is the corresponding quantity. 
The integrals which will represent the form of the surface at any time, when the disturbanco is cnusod by impulsivo pressures applied over a limited extent of surface, are gencrally of slightly different form to those arising from the cause considered in this paper; but similar methods may be used to approximato to their values. The forms obtained for the surface and the general laws which the motion follows are, as is to bo expected, precisely similar to the results obtrined here.

On a cortain Atomic IIypothesis. By Prof. Kari. Pearson.

[Rcal Nov. 8th, 1888.]

1. In a papor written in 1883 , and read beforo tho Cambrilgo Philosophical Socict.y in 1855 (Camb. 1'hil. I'rans., xiv., Part II., pp. 71-120), I snpposed, as a first approximation, that the ultimato constituents of natter might be treated as splereacal bodies capable of surfaco vibration and pulsation. I then ondeavoured to show that the free and forced vibrations of such atoms were sufficient to produce in a fluid ether many of the phenomena of chemical aflinity, coliesion, and gravitation. 'lhe assumptions really made wero:

(i.) The pressures produced on the surface of one atom by the vibrations of another are, at any rate to a first approximation, identical with those which would be produced were the ether " perfiect fluid.

(ii.) 'l'he nltimite atom inily, to a first approximation, be treated as a vibrating sphere.

In the course of my work, I did not pre-suppose any special inter'nal structure for the spherical atom, bcyond a capacity for absorbing ener'gy in the form of surface vibrations. I also gave sorne reasons for the belief that a sinple pulsation must play a more important part than polar vibiations within tho sphere of inter-atomic influcnce. Further, the moro complex molecule, which forms the batsis of the 